\title{
Tres aproximaciones a la complejidad
}

Fecha de recepción: 20.08.2010

Fecha de aceptación: 28.01.2011

Alfredo Díaz Mata

Facultad de Contaduría y Administración, Universidad Nacional Autónoma de México adiazm@fca.unam.mx

\section{Resumen}

En este artículo se propone agrupar en tres vertientes las distintas formas en las que se está abordando actualmente el tema de la complejidad: 1) desde las ciencias "duras", 2) como universalidad y 3) desde las ciencias sociales; en esta última aproximación se ubica la relación de la complejidad y la administración. Se exponen sus principales ideas y propuestas para identificarlas y mostrar que son claramente distinguibles y que, al mismo tiempo, tienen también numerosas convergencias. El trabajo intenta despejar algunas de las confusiones que se dan alrededor del tema de la complejidad y dar una perspectiva global de esta activa área actual de investigación y aplicación. Se muestra cómo es que las aproximaciones actuales a la complejidad constituyen un área de investigación que está en proceso de consolidación y que está dando lugar a avances y aplicaciones en muy distintas perspectivas, tanto en las ciencias duras, como en las ciencias sociales y las humanidades, al tiempo que se proponen traspasar las barreras disciplinarias y las que separan a estos tres campos del saber.

Palabras clave: complejidad, ciencias "duras", universalidad, ciencias sociales. 


\title{
Three approaches to complexity
}

\begin{abstract}
This text advances that the different approaches being taken by those who currently study complexity can be divided three ways: 1) from the perspective of the "hard sciences", 2) as a universality, and 3) from the point of view of the social sciences, and the topic of Management and Complexity is placed under this third approach. The paper tries to clarify some of the confusions presently surrounding complexity and presents a global perspective of this active area of research and application. The main ideas and propositions of these approaches are presented in order to show that they are clearly distinguishable and that, at the same time, they converge in several ways. It is shown the way these three approaches to complexity constitute a research area undergoing a consolidation process that is producing progresses and applications in the hard sciences as well as in the social sciences and the humanities and that, at the same time, is trying to surpass disciplinary boundaries and those separating these three areas of Knowledge.
\end{abstract}

Keywords: complexity, "hard sciences", universality, social sciences.

\section{Tres aproximaciones a la complejidad ${ }^{1}$}

Complejidad es una palabra de moda. Se habla de ella en todo tipo de ambientes: científicos, académicos, de difusión e informales. Y, aparte de que, por supuesto, la palabra sigue teniendo su significado común de "complicado", es cada vez más frecuente su uso en ámbitos más especializados y con significados igualmente particulares, como se expone aquí.

Este artículo se propone distinguir tres aproximaciones a la complejidad con lo que se pretende despejar algunas de las confusiones que se dan alrededor de este tema, así como dar una perspectiva global de esta activa área actual de investigación y aplicación. La propuesta básica consiste en considerar que las diversas temáticas que se están abordando en la actualidad con relación a la complejidad pueden clasificarse dentro de tres aproximaciones principales: la complejidad desde las

\footnotetext{
${ }^{1}$ Agradezco los enriquecedores comentarios de los miembros del Seminario de Complejidad de la División de Investigación de la Facultad de Contaduría y Administración de la Universidad Nacional Autónoma de México: Luis Antonio Cruz Soto, Mónica González Hernández, Yazmín Hernández Juárez, José Silvestre Méndez Morales, Fernando Paz Sánchez y Paola Selene Vera Martínez, a la vez que asumo completa responsabilidad por lo que aquí se expresa.
} 
ciencias "duras", la complejidad como universalidad y la complejidad desde las ciencias sociales.

Carlos Maldonado dice en Visiones sobre la complejidad (citado por Sotolongo, 2006, p. 43) que "en los estudios sobre la complejidad pueden distinguirse tres líneas de comprensión del asunto: a) la complejidad como ciencia (el estudio de la dinámica no lineal en diversos sistemas concretos); b) la complejidad como método de pensamiento (la propuesta de un método de pensamiento que supere las dicotomías de los enfoques disciplinarios del saber y que consiste básicamente en el aprendizaje del pensamiento relacional); y c) la complejidad como cosmovisión (la elaboración de una nueva mirada al mundo y al conocimiento que supere el reduccionismo a partir de las consideraciones holistas emergentes del pensamiento sistémico)". Como se verá, la complejidad como ciencia corresponde, más o menos, a lo que aquí se plantea como la aproximación desde las ciencias duras, en tanto que la interdisciplinariedad (la visión b) está presente en las tres aproximaciones que aquí se proponen. Por su parte, la visión c, la complejidad como cosmovisión, se corresponde aproximadamente con la aproximación aquí expuesta como la "universal". Finalmente, en este trabajo se propone una aproximación a la complejidad desde las ciencias sociales, que no está presente en el planteamiento de Maldonado y que incluye una subcategoría que se ha denominado "la complejidad disciplinaria", que cubre los temas de la relación de la complejidad con diversas disciplinas sociales como complejidad y finanzas, complejidad y economía y una que es de particular importancia para la administración y que se denomina, precisamente, complejidad y administración.

Por cuestiones de espacio, este tema especialmente importante para la administración se abordará con el detalle que merece en otro trabajo dedicado a ello. Por lo pronto, en este artículo, se abordan las tres aproximaciones que se proponen a la complejidad y que, tal como se ha mencionado y se espera, permitirán, además de despejar algunas de las confusiones que se dan alrededor de este tema y de dar una perspectiva global de esta activa área actual de investigación y aplicación, ubicar en su contexto más amplio el área de estudio de la complejidad y la administración.

Se exponen a continuación las tres vertientes para abordar la complejidad que, como se propone aquí, son claramente distinguibles y que, al mismo tiempo, tienen también numerosas convergencias. Se comienza en los párrafos siguientes con una breve exposición de algunas de esas confusiones, junto con los elementos princi- 
pales que, según quien esto escribe, distinguen a esas tres aproximaciones para, en las secciones siguientes, exponer resumidamente sus principales ideas, conceptos y propuestas.

Las confusiones sobre lo que "complejidad" significa se deben a que ésta es un área del conocimiento sumamente amplia y diversa, que se encuentra todavía en construcción y que está en constante crecimiento y esto es patente, entre otras cosas, en la proliferación de centros de investigación dedicados al estudio de la complejidad que abordan este tema con enfoques distintos, como se puede apreciar en los planteamientos de sus objetivos. El Santa Fe Institute en Nuevo México, Estados Unidos, fundado en 1984, es "una comunidad de investigación transdisciplinaria que está expandiendo los límites de la comprensión científica [y cuyo] objetivo es descubrir, comprender y comunicar los principios comunes fundamentales de los sistemas complejos físicos, computacionales, biológicos y sociales que subyacen a muchos de los problemas más profundos que enfrentan actualmente la ciencia y la sociedad" (www.santafe.edu/, 21 ene 2010). Destaca en este planteamiento la búsqueda de los "principios comunes fundamentales de los sistemas complejos" y, también, la mención de la "transdisciplinariedad", tema también muy de moda al que se vuelve más adelante.

En México, específicamente en la Universidad Nacional Autónoma de México (UNAM), se creó en 1985 el Departamento de Sistemas Complejos en el Instituto de Física y, posteriormente, a finales de 2008, el C3 (Centro de Ciencias de la Complejidad) que es "un punto de encuentro para más de cincuenta investigadores y cuarenta estudiantes de posgrado de diversos institutos, facultades, y universidades [...] cuya misión es realizar investigación científica transdisciplinaria de frontera en las ciencias de la complejidad, creando un espacio en donde expertos de muy diversas áreas puedan interactuar y contribuir a la solución de problemas trascendentes y de importancia nacional. Es también misión del Centro formar científicos entrenados en el trabajo transdisciplinario en equipo, y en el fortalecimiento de los métodos modernos asociados a la ciencia computacional" (www.nucleares, 21 ene 2010), de nuevo surge la complejidad, ahora en la forma de "ciencias de la complejidad" y la transdisciplinariedad. Se han creado instituciones similares en muchos países del mundo.

Las confusiones han sido propiciadas también por la proliferación de programas académicos relacionados con la complejidad que al igual que sucede con los centros de investigación, abordan la complejidad desde perspectivas diferentes como, 
por ejemplo, los que se imparten en el anteriormente citado Instituto Santa Fe y una Maestría en Ciencias de la Complejidad en la Universidad Warwick, en Coventry, Inglaterra, una Cátedra de Complejidad y Transdisciplinariedad Educativa Convenio "Andrés Bello", en Bolivia. En México se imparte actualmente una maestría en Dinámica No Lineal y Sistemas Complejos en la Universidad Autónoma de la Ciudad de México. A las confusiones contribuyen asimismo las numerosas publicaciones que existen actualmente sobre el tema. Una búsqueda de libros con el título de "complexity" en Amazon.com arroja un total de 5,908 resultados (26 julio 2010), en tanto que una búsqueda de "complejidad" en la base bibliográfica de la Universidad Nacional Autónoma de México arroja 108 resultados. En www.comdig.org/resources.php (23 febrero 2010) se listan 14 revistas sobre, o relacionadas con, complejidad, entre los que se encuentran Advances in Complex Systems; Chaos: An Interdisciplinary Journal of Nonlinear Science; y Emergence: A Journal of Complexity Issues in Organizations and Management. Entre estas numerosas publicaciones se encuentra una edición especial de Organization Science, la cual dedicó su edición de mayo-junio de 1999 a "las aplicaciones de la teoría de la complejidad a la ciencia de la organización” (www.jstor.org/pss/2640329).

Además de lo anterior, quienes están trabajando actualmente con seriedad sobre el tema de la complejidad hacen que focalizarlo sea adicionalmente difícil porque se habla tanto de ciencia de la complejidad, en singular, como de ciencias de la complejidad, en plural y, por otro lado, se habla también de "teoría de la complejidad", "teoría de los sistemas complejos" y "ciencia de los sistemas complejos". Igualmente, se habla de "sistemas complejos" y de "pensamiento complejo" y se mencionan, asimismo, abordamientos de la complejidad desde diversas disciplinas a las cuales se les agrupa en las "ciencias duras" y las "ciencias sociales y las humanidades". Por ejemplo, Melanie Mitchell (2009, xiii) habla, sin mayor explicación, de ciencia y de ciencias de la complejidad en la misma página. Neil Johnson (2007, ix) afirma que "no tenemos aún una "teoría" de la complejidad completamente desarrollada", al tiempo que Rolando García (2006, pp. 21-39 y, en general, todo el libro) expone, con considerable detalle, lo que él llama "Teoría de los Sistemas Complejos".

Ante este panorama, como rebasa el propósito de este estudio, se pasa por alto la discusión sobre si se trata de una teoría o una ciencia y la que se refiere a si es una sola o son más, dado que no existe una definición formal universalmente aceptada de complejidad, ni de ciencia o teoría de la complejidad, ni de sistemas complejos y se procede, en lo que resta del trabajo, a exponer las tres vertientes señaladas para 
abordar la complejidad, las cuales son claramente distinguibles y que, al mismo tiempo, tienen también numerosas convergencias.

\section{La complejidad desde las ciencias duras}

Como antecedentes de esta visión de la complejidad se suelen citar los avances científicos de los últimos 500 años y se destacan las aportaciones de Galileo Galilei (1564-1642) y de Isaac Newton (1643-1727). John Gribbin (2004, pp. 4-5) afirma que fue Galileo quien "estableció los principios del método científico de investigación, el cual implica comparar teorías (o modelos) con el resultado de observaciones y experimentos", al tiempo que afirma que el "más grandioso descubrimiento científico del siglo XVII, y quizá de todos los tiempos [fue] la ley de la gravitación universal de Newton" (2004, p. 7). Aquí vale la pena recordar que fueron Isaac Newton y Wilhelm Leibniz (1646-1716) quienes desarrollaron por separado y casi al mismo tiempo el cálculo diferencial e integral que es una herramienta que ha sido fundamental en los avances de la física, en otras áreas de investigación y, en tiempos más recientes, en muchos de los desarrollos actuales de la teoría de los sistemas complejos. Esta manera de hacer ciencia a través de teorías verificables experimentalmente es la que ha permitido enormes avances en muchas áreas del conocimiento y en particular en las "ciencias duras": física, química, matemáticas, biología, etc. Entre las principales aportaciones de todos los científicos de estas áreas están la física cuántica, las leyes de la termodinámica, la teoría de la relatividad, la teoría de las estructuras disipativas, la teoría general de los sistemas, la teoría de la información y la cibernética, la biología molecular, los fractales y, ahora, las teorías del caos y de los sistemas complejos.

En los apartados siguientes se abordan algunas de las principales ideas (e, incluso, teorías) sobre las que se ha desarrollado esta manera de abordar la complejidad y que giran en torno del concepto de los sistemas complejos, ${ }^{2}$ la teoría del caos y los fractales, la interdisciplinariedad, y la propia complejidad.

\footnotetext{
${ }^{2}$ Aunque este enfoque "duro" de la complejidad gira en buena medida en torno al concepto de los "sistemas complejos", se decidió aquí no utilizar esta frase como calificativo para identificarla dado que, como se menciona antes, el enfoque "social" tipificado a través de las propuestas de Rolando García propone también una "teoría de los sistemas complejos" con un sentido considerablemente diferente.
} 
Algunos de los principales conceptos de la teoría de los sistemas complejos

"Un sistema complejo es un sistema en el cual grandes redes de componentes sin un control central y con reglas simples de operación dan lugar a una conducta colectiva compleja, a procesamiento sofisticado de información y a adaptación a través de aprendizaje o evolución" (Mitchell, 2009, p. 13). De esta definición se desprenden, entre otras, dos características muy importantes de los sistemas complejos: la autoorganización y la emergencia. La autoorganización se refiere a que los elementos del sistema se organizan sin que haya dirigentes o controladores centrales, en tanto que la emergencia se refiere a que se produce comportamiento complejo a partir de esas interacciones no dirigidas.

Algunos ejemplos de sistemas complejos que se ajustan a esa definición son colonias de hormigas que se autoorganizan "para buscar alimento, para responder en formas simples a las señales químicas de otras hormigas de la colonia, para atacar invasores, etc." (Mitchell, 2009, p. 4); el cerebro, en el cual un conjunto muy numeroso de neuronas, mediante mecanismos relativamente simples de comunicación entre ellas (pulsos eléctricos) dan lugar a la elaborada conducta global de la mente; y el sistema inmunológico de los animales, formado por muchos tipos diferentes de células que operan en forma conjunta, sin un control central, para combatir invasores nocivos (Mitchell, 2009, pp. 6-9). "En estos sistemas, los agentes que residen en una escala comienzan a producir comportamientos que yacen en una escala superior a la suya: las hormigas crean colonias, los habitantes de una ciudad crean barrios, un software de reconocimiento de patrones simple aprende a recomendar libros: La evolución de reglas simples a complejas es lo que llamamos «emergencia»" (Johnson, 2001, p. 19).

Sobre la autoorganización vale la pena destacar que se trata de un proceso y que estos procesos de autoorganización conducen a una complejidad creciente. El desarrollo de la vida es un magnífico ejemplo de cómo la autoorganización y la evolución han dado lugar a organismos cada vez más complejos. Además de los temas expuestos, ya se habían también mencionado como temas estrechamente relacionados con la teoría de los sistemas complejos la interdisciplinariedad y la teoría del caos, así como los fractales. En los apartados siguientes se abordan estas cuestiones. 


\section{Teoría del caos}

El estudio actual del caos proviene de la teoría de los sistemas dinámicos la cual, a su vez, cuenta entre sus antecedentes a autores como Copérnico, Galileo y, en especial, a Newton con sus estudios sobre el movimiento de los cuerpos celestes y cuya culminación son sus tres famosas leyes del movimiento: ley de la inercia, ley de la fuerza y ley de acción y reacción, que "son los fundamentos de la dinámica: explican el movimiento de los planetas, y de todo, en términos de las nociones básicas de fuerza y masa" (Mitchell, 2009, p. 19).

Esta misma autora afirma que "La teoría de los sistemas dinámicos no lineales ha estado recientemente en boga en la ciencia popular debido a los fascinantes resultados que provienen de uno de sus resultados intelectuales, el estudio del caos" (Mitchell, 2009, p. 16). Esta teoría de los sistemas dinámicos aborda el estudio de sistemas que cambian (dinámicos) en el tiempo y/o el espacio y que son no lineales en el sentido de que los cambios que se producen en el sistema no son proporcionales a las causas; en otras palabras, causas pequeñas o grandes no necesariamente producen efectos pequeños o grandes, respectivamente, sino que causas pequeñas pueden producir resultados enormes y viceversa.

Uno de los ejemplos más sencillos y utilizados para ilustrar los sistemas dinámicos no lineales y el caos es lo que se conoce como la ecuación logística:

$$
x_{i}=r x_{i-1}\left(1-x_{i-1}\right)
$$

Revisando esta sencilla ecuación se puede ver que cada valor de la variable $\mathrm{x}$ depende de una constante $\mathrm{r}$ y del valor anterior de la x. La ilustración del caos se hace iterando esta ecuación, es decir, resolviéndola repetidamente y alimentando en cada repetición (iteración) el valor de x obtenido en la iteración anterior. Para iniciar las iteraciones se requiere un valor inicial de $\mathrm{x}$, que se denota como $\mathrm{x}_{0}, \mathrm{y}$ un valor fijo para r. Si se hace esto, se encuentra que ${ }^{3}$ :
Con $0<r \leq 1$ $x_{i} \rightarrow 0 \quad$ sin importar el valor de $\mathrm{x}_{0}$,
Con $1<r \leq 3$ $x_{i} \rightarrow$ a un valor fijo, superior a cero y menor a $2 / 3$

\footnotetext{
${ }^{3}$ Aunque existen numerosos textos que explican el comportamiento de esta ecuación logística, se sugiere consultar la magnífica y clara exposición que presenta Johnson, 2007, pp. 46-50.
} 
Con $3<r \leq \sim 3.569946 \quad x_{i} \rightarrow$ muestra un comportamiento periódico que se duplica desde $r$ ligeramente superior a 3 , hasta cuando el valor es aproximadamente, 3.569946, punto a partir del cual el comportamiento de $x_{i}$ se vuelve caótico. A este punto, que marca la separación entre el comportamiento regular, fijo o periódico, y el caótico, se le denomina "punto crítico" y se le conoce también como punto de Feigenbaum, que es igual a 3.5699456. En la figura 1 se muestra una gráfica que ilustra este comportamiento.

Figura 1

Diagrama de bifurcación para el mapa logístico

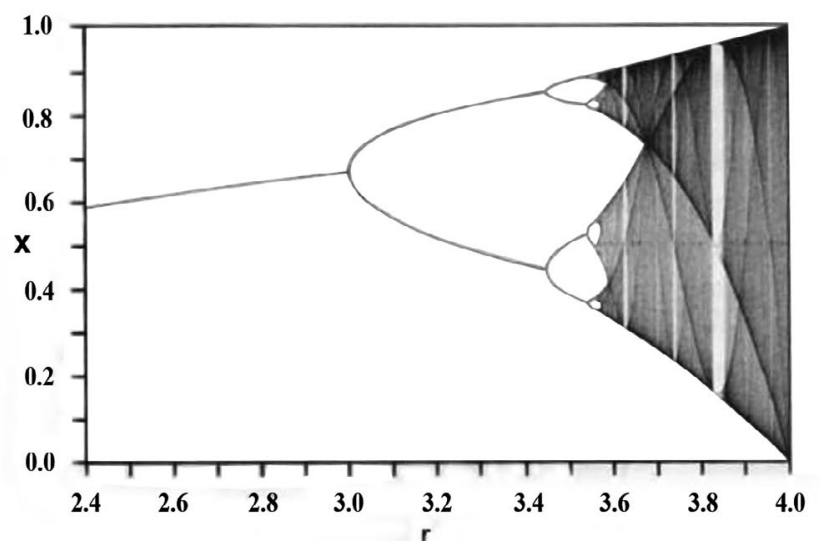

Fuente: Mitchel, 2009, p. 34.

Este comportamiento de la ecuación logística ilustra varios puntos interesantes. Por un lado, ejemplifica claramente lo que en teoría del caos (y en la teoría de los sistemas complejos) se conoce como "atractor". Y hay aquí tres tipos. Como con $0<r \leq 3$, $\mathrm{x}_{\mathrm{i}}$ se va a valores fijos entre 0 y $0.6666 \ldots$, se dice que se tiene un atractor, precisamente, de punto fijo, un número específico. Por su parte, como con $3<r \leq \sim 3.569946$, $\mathrm{x}_{\mathrm{i}}$ muestra un comportamiento periódico que se duplica, se dice que se tiene un atractor periódico. Finalmente, como a partir de ese valor aproximado de 3.569946 el comportamiento de esta ecuación logística se vuelve caótico se tiene, entonces, un atractor caótico o, como también se le conoce, un atractor extraño. Sin embargo, se sabe que otra característica de muchos sistemas dinámicos no lineales es que "siguen una ruta de duplicación de periodo al caos, característica universal de los sistemas caóticos" (figura 1) aunque, por otra parte, como afirma Johnson (2007, p. 48), "El caos puede ser visto simplemente como un patrón periódico cuyo periodo es tan prolongado que nunca se repite". 


\section{Fractales}

La relación entre caos y fractales la establece, en términos considerablemente sencillos Érdi (2008, p. 20) cuando afirma que "los atractores extraños tienen estructura fractal", tema que también ilustra Johnson (2007, p. 51) cuando ejemplifica que se puede construir un "polvo fractal" dividiendo sucesivamente en dos a una línea recta (figura 2).

Figura 2

Producir un polvo fractal

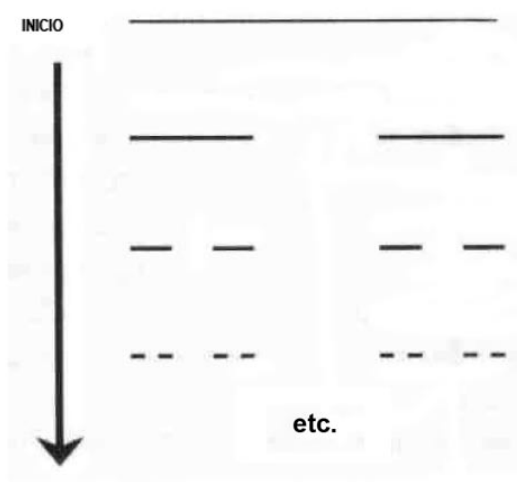

Fuente: Johnson, 2007, p.51

Érdi (2008, p. 60) afirma también que "se considera que los procesos caóticos son generadores de complejidad" y los fractales son un buen ejemplo de esto.

Los fractales han probado ser una excelente manera de describir una gran cantidad de formas, tanto naturales como artificiales. Benoit Mandelbrot trabajó abundantemente este tema (véase, por ejemplo, su obra de 1977, La geometría fractal de la naturaleza y es famoso el "conjunto de Mandelbrot", que es un ejemplo clásico de la belleza intrínseca de estas formaciones y que se reproduce en la figura 3. 
Figura 3

El conjunto de Mandelbrot

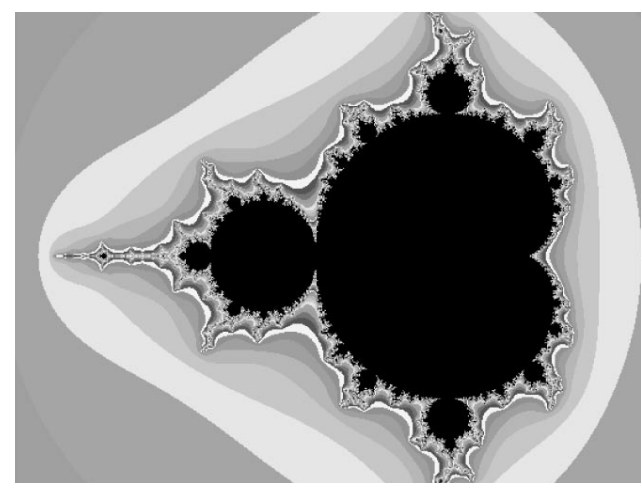

Aunque no existe una definición estática, sencilla y universalmente aceptada de los objetos denominados "fractales", se puede hacer una aproximación mediante algunas de sus características (Díaz Mata, 1996):

1. Son autosimilares ${ }^{4}$ porque conforme se les observa más de cerca, más detalles muestran y estos acercamientos tienen la misma forma que el objeto completo. En otras palabras, se trata de curvas, superficies o sólidos que son independientes de la escala. Esta característica se puede observar claramente en el conjunto de Mandelbrot.

2. Muestran elevada sensibilidad a las condiciones iniciales. Esta característica se puede ilustrar en la construcción de fractales a partir de la iteración de ecuaciones muy simples (como la famosa ecuación logística) en donde se obtienen resultados muy diferentes a partir de valores iniciales muy ligeramente distintos. Determinado valor puede conducir a comportamientos (atractores) fijos, regulares o periódicos o simétricos, en tanto que otro valor, apenas diferente, puede conducir a resultados muy complejos o caóticos.

3. Tienen dimensión fraccionaria y no entera, como euclideanamente se maneja. Se puede dar una idea intuitiva de esto pensando que una línea

\footnotetext{
${ }^{4}$ Benoit B. Mandelbrot hace una distinción entre fractales autosimilares y fractales autoafines que es importante para el análisis de precios bursátiles, pero que escapa al alcance de este artículo. Véase Fractals and Scaling in Finance, Springer, EUA, 1997, pp. 149-151
} 
tiene dimensión 1, en tanto que una superficie tiene dimensión 2, pero parecería evidente que una curva que se retuerce hasta casi llenar un plano tiene dimensión superior a 1, pero menor de 2; es decir, tiene dimensión fraccionaria o fractal.

\section{Sistemas complejos e interdisciplinariedad}

Es la propia Mitchell (2009, p. 4) quien plantea que el tema de los "sistemas complejos [es] un área interdisciplinaria de investigación que busca explicar la forma en la que grandes números de entidades relativamente simples se organizan entre sí, sin el beneficio de un controlador central, para convertirse en un todo colectivo que crea patrones, utiliza información y, en algunos casos, evoluciona y aprende". Se repiten aquí varias de las características de los sistemas complejos que ya se revisaron antes pero, además, se introduce la idea de un abordamiento interdisciplinario para los sistemas complejos. Y, en la misma página, afirma que "El campo de la complejidad es, en sí mismo, un entramado de muchos campos diferentes".

En la enciclopedia de d'Alembert y Diderot, cuya primera edición constó de diecisiete tomos, "se hace una clasificación exhaustiva de las artes, las ciencias y los oficios, con lo que quedan establecidas las fronteras entre las diversas disciplinas" 5 lo cual, a su vez, conlleva en la actualidad un "factor institucional, de gremios académicos — cuando no de mafias— de intereses y de poder". En contraposición a la postura enciclopedista, en 1970, la Organización para la Cooperación y el Desarrollo Económicos convocó a una reunión para tratar el tema y "pronto muchas universidades crearon áreas o centro de estudios interdisciplinario". Actualmente, las tres corrientes para abordar la complejidad que se distinguen en este trabajo coinciden en que la interdisciplinariedad es imprescindible para su estudio.

Antes de concluir esta breve exposición de la perspectiva de la complejidad, desde las ciencias duras, conviene añadir aquí que son básicamente tres los enfoques que se están aplicando desde esta corriente para construir una teoría o ciencia de los sistemas complejos y que se podrían caracterizar, por una parte, como estrategias de modelización de sistemas desde las matemáticas, con ecuaciones diferenciales y en diferencias, análisis, topología y teoría de redes. Un segundo enfoque es el de la ciencia de la computación, con base en modelos basados en agentes artifi-

${ }^{5}$ Los argumentos y citas de este párrafo se basan en Köppen, Mansilla y Miramontes (2005, p. 4-12). 
ciales y en autómatas celulares, entre otros; por cierto, vale la pena no pasar por alto que, sin las computadoras, el avance que se ha dado en el análisis de sistemas dinámicos complejos simplemente no habría sido posible (Érdi, 2008, cap. 9). El tercer enfoque es el experimental; en una amplia variedad de disciplinas, como la física, la ingeniería, la ecología, la epidemiología, la economía, la sociología, por citar algunas, se han realizado experimentos que confirman muchas de las ideas desarrolladas en la teoría de los sistemas complejos.

Y, finalmente, algunos ejemplos de los problemas que se están abordando desde esta perspectiva: pronósticos de temblores, de ataques epilépticos y de desplomes bursátiles (Érdi, 2007, pp. 328-352), "hacia una teoría unificada del cerebro-mente y la computadora" (Érdi, 2007, pp. 289-304). "La NASA está investigando la posibilidad de que se pueda utilizar un conjunto de robots relativamente simples para explorar la superficie de un planeta de manera rápida y eficiente" o "máquinas que se puedan diseñar a sí mismas, permitiendo que las máquinas individuales se adapten y evolucionen por sus propios medios" (Johnson, 2007, pp. 6-9), o la posibilidad de aprender cosas útiles para el diseño de cadenas de abastecimiento en el comercio al menudeo, a partir de las líneas de provisión de nutrientes en hongos (Johnson, 2007, p. 16). O, a través del uso de "ingeniería de poblaciones", utilizar numerosas aletas pequeñas en aviones que, mediante la competencia entre sí, permitirían controlar algunas operaciones del aparato, o la introducción de células en tumores cancerosos para que, de nuevo mediante la competencia entre todas las células, dirijan al tumor hacia su propia extinción o, en finanzas, la introducción de agentes en mercados financieros para evitar turbulencias, de nuevo, orientando la competencia entre los agentes (Johnson, 2007, pp. 92-95).

\section{La complejidad como universalidad}

Es posible que el representante más conspicuo de esta corriente sea Edgar Morin, pensador francés, que tiene una copiosa obra sobre el tema del pensamiento complejo que incluye, entre otros, un libro titulado Introducción al pensamiento complejo $(1990)^{6}$ y seis tomos referidos a la metodología para abordar con nuevos fundamentos "el principio organizador del conocimiento [ya que] lo que es vital hoy, no es solamente aprender, no solamente reaprender, no solamente desaprender, sino reorganizar nuestro sistema mental para reaprender a aprender". Y dice

\footnotetext{
${ }^{6}$ Vale la pena anotar que, aunque la obra consultada fue publicada en 2007, la edición francesa original es de 1990, y es el año con el que se identifican las obras en la bibliografía y en estas referencias.
} 
también que lo que enseña a aprender es el método, pero "No aporto el método, parto a la búsqueda del método" (Morin, 1981, p. 35).

Este autor plantea que "ninguna ciencia ha querido conocer la categoría más objetiva del conocimiento: la del que conoce. Ninguna ciencia ha querido conocer su origen cultural" (1981, p. 24), con lo que se acerca, como se verá, a la corriente del enfoque social que se revisa más adelante: es necesario comprender las interpretaciones y sesgos que quien conoce incluye en el conocimiento.

Una de las principales ideas de la propuesta de Morin gira "en torno a la intención de articular lo físico con lo biológico y ambos con lo antropológico, psicológico y mitológico", según lo expresa Marcelo Pakman en la introducción a Morin (1990, p. 12). Uno de los planteamientos básicos de Morin es que "la complejidad es el desafío, no la respuesta" (2007, p. 143) o, en otras palabras, "La complejidad es una palabra problema y no una palabra solución” (2007, p. 22). En el prólogo de esta obra, Morin abunda sobre la complejidad y afirma que lo que se requiere es un pensamiento complejo que no es fácil de justificar sino que ha de imponerse progresivamente a lo largo de un camino en el cual aparecerán, ante todo, los límites, las insuficiencias y las carencias del pensamiento simplificante, es decir, las condiciones en las cuales no podemos eludir el desafío de lo complejo" (2007, p. 22). Abordar la complejidad es, así, un proceso que es, entre otras cosas, largo, difícil e interminable y que, además, "incluye la imperfección porque incluye la incertidumbre, y el reconocimiento de lo irreductible" (2007, p. 143).

Acerca de lo irreductible, Morin afirma, con enorme tino, según este ensayista, que la complejidad debe manejar lo que se puede racionalizar y lo que no, ya que "la racionalidad tiene como misión dialogar con lo irracionalizable" (2007, p. 34).

Al igual que en la visión desde las ciencias duras, Morin tampoco deja de observar la existencia de interacciones que, para aquella, dan lugar a la no linealidad y el caos: “¿Qué es la complejidad? A primera vista, es un fenómeno cuantitativo, una cantidad extrema de interacciones e interferencias, entre un número muy grande de unidades" (2007, p. 59), y "la complejidad es, efectivamente, el tejido de eventos, acciones, interacciones, retroacciones, determinaciones, azares, que constituyen nuestro mundo fenoménico" (2007, p. 32).

Y, a las rachas de comportamiento ordenado seguidas de rachas de comportamiento desordenado que se señalaron como características de los sistemas complejos, 
Morin afirma que "La complejidad es la dialógica orden/desorden/organización" (2007, p. 145).

Morin aborda también la cuestión de la necesidad de resolver los abundantes problemas que la realidad plantea, ya que "los seres humanos, la sociedad, la empresa, son máquinas no triviales" (2007, p. 116) y, por ello, es necesario estar conscientes de que el pensamiento simple resuelve los problemas simples y el pensamiento complejo, aunque no resuelve por sí mismo los problemas complejos, "constituye una ayuda para la estrategia que puede resolverlos" (2007, p. 118).

En el terreno de la práctica, una de las actividades en las que ha participado activamente Edgar Morin ha sido la transformación de la educación. Existe una Cátedra Itinerante UNESCO "Edgar Morin" para el Pensamiento Complejo (http://ciuem. org/?q=node/1) que, como parte de sus diversas actividades, ha producido un texto (Morin, Ciurana y Motta, 2006), que se titula "Educar en la era planetaria" y en el que se abordan, entre otros temas, el método como estrategias para el conocimiento y la acción en un camino que se piensa (parte I) y los desafíos de la era planetaria como el posible despertar de una sociedad-mundo (parte III). Otro trabajo de Morin (1999), que escribió a solicitud de la UNESCO (Organización de las Naciones Unidas para la Educación, la Ciencia y la Cultura), lista y analiza los siete saberes necesarios para la educación del futuro: enseñar 1) cómo es que se conoce, 2) un conocimiento capaz de abordar los problemas pertinentes, globales y fundamentales, 3) la condición humana, el ser humano como entidad a la vez física, biológica, síquica, cultural, social e histórica, 4) la identidad terrenal, el planeta como una unidad interrelacionada e históricamente construida, 5) enfrentar las incertidumbres, 6) la comprensión mutua entre los seres humanos y 7) la ética del género humano.

Existen otros autores que, aunque sea aproximadamente, coinciden con algunas de las ideas de Morin, por lo que se podrían incluir en esta corriente "universal". En el siguiente apartado, en calidad de ejemplo, se presentan brevemente las propuestas de Fritjof Capra, en donde se destacan sus relaciones con las propuestas del propio Edgar Morin y con los conceptos desarrollados por la corriente de la complejidad desde las ciencias duras. 
Fritjof Capra: la integración de las dimensiones biológica, cognitiva y social

En el prefacio de su libro Las conexiones ocultas, Fritjof Capra (2002, p. 17) afirma que se propone "presentar un marco conceptual que integre las dimensiones biológica, cognitiva y social de la vida" y divide la primera sección del texto en tres capítulos titulados: La naturaleza de la vida, La mente y la conciencia, y La realidad social, respectivamente, y dedica la segunda parte del libro a las aplicaciones del marco teórico expuesto en esos tres primeros capítulos.

Los planteamientos que expone en esa primera sección conducen a lo que de manera muy resumida podría exponerse de la siguiente forma: la estructura material de los seres vivos está organizada en forma de una red autogenética (autopoiética) que conforma un sistema abierto que opera lejos del equilibrio y que da lugar a procesos que constituyen sistemas cognitivos, en los que el proceso de cognición está íntimamente ligado al patrón de autopoiesis. Este planteamiento integra, la "síntesis de la nueva comprensión científica de la vida" de Capra (p. 104) y parte de aquí para incluir la dimensión social con lo que, propone, se puede llegar a "una comprensión sistémica de la realidad social" (p. 115), que "sólo podrá emerger cuando se combinen los conceptos de la dinámica no lineal con las ideas de esos campos de estudio [teoría social, filosofía, ciencia cognitiva y antropología, entre otras] ... [ya que] Una red social es ... un patrón no lineal de organización, por lo que es muy probable que las ideas y los conceptos desarrollados en la teoría de la complejidad, como la retroalimentación y la emergencia, sean también relevantes en el contexto social" (p. 116).

En este muy apretado tratamiento de las ideas de Capra se puede observar su cercanía con la visión universal de Morin pero, al mismo tiempo, su propuesta de utilizar "las ideas y conceptos desarrollados en la teoría de la complejidad" que, para puntualizar lo que aquí se ha dicho antes, diría "la teoría de la complejidad desde las ciencias duras", ya que esos conceptos de redes, retroalimentación y emergencia que menciona Capra aparecen también en ese campo, junto con otros que tienen el mismo origen, pero que Capra no menciona y que podrían tener también aplicación en su propuesta de "una comprensión sistémica de la realidad social", como podrían ser los de autoorganización y caos, entre otros. 


\section{Complejidad desde las ciencias sociales}

Con base fundamentalmente en la epistemología genética de Jean Piaget, Rolando García propone una "epistemología constructivista", o "constructivismo", tomando en cuenta desarrollos posteriores a Piaget (2000, p. 26), a partir de tres principios generales que rigen la evolución del sistema cognoscitivo: 1) continuidad de los procesos, 2) continuidad de los mecanismos y 3) desarrollo por reorganizaciones sucesivas (2000, p. 125).

Abundando sobre lo anterior, este autor, García, expone que "los procesos constructivos [del conocimiento] consisten en un juego dialéctico que conjuga la organización de las propias acciones con la organización de los "datos" del mundo exterior provenientes de las interacciones sujeto/objeto" (2000, p. 110) y que, al mismo tiempo, "se van diferenciando elementos que aparecían como "un todo", y se van integrando datos con interpretaciones previas que aparecían como independientes" (2000, p. 110). Y considera que estos procesos de diferenciación e integración que aparecen desde las etapas iniciales de la construcción del conocimiento, es decir desde el nacimiento mismo, "pero con creciente necesidad de tomar en cuenta los procesos biológicos que lo preceden" (2000, p. 25), acaban por constituirse "en un mecanismo fundamental en la organización del conocimiento" (2000, p. 210).

Así, aparece como evidente la continuidad de los procesos, que son, entonces, un juego dialéctico que combina la organización de las acciones de la persona con la organización de los "datos" provenientes del mundo exterior y que provienen de la interacción de la persona con éstos. Y, a la vez, se presenta también como evidente la continuidad de los mecanismos de organización del conocimiento a través de reorganizaciones sucesivas, producidas mediante las diferenciaciones e integraciones mencionadas.

Y resume García que el desarrollo del conocimiento consiste en un doble proceso constructivo (2000, p. 112):

La organización de las propias actividades del sujeto, que comienza con la coordinación de sus acciones, continúa con el desarrollo de los mecanismos constructivos del conocimiento y culmina con la lógica, es decir, en las formas deductivas y los reforzamientos. 
La organización del material empírico, que comienza con asignación de significados, continúa con comparaciones que conducen a correspondencias y transformaciones elementales y culmina con la interpretación de fenómenos estableciendo relaciones causales.

Como parte de una "teoría de la equilibración", aparece un elemento adicional importante en la epistemología constructivista que propone Rolando García, el cual consiste en la existencia de dos tipos de procesos de construcción de conocimiento, "con dinámicas particulares" $(2000,108)$. Por un lado, habría las "Fases organizativas, que en niveles superiores serán fases estructurantes" y las "Fases organizadas, que corresponden a periodos donde se pueden reconocer estructuras más o menos estabilizadas (fases estructuradas)" (2000, p.108), que es claro que provienen de las reorganizaciones sucesivas que se dan en el proceso de construcción del conocimiento y que tienen un evidente paralelismo con las rachas de comportamiento ordenado seguidas de rachas de comportamiento desordenado que se listaron como una característica frecuentemente presente en los sistemas complejos contemplados desde las ciencias duras.

De hecho, García (2006, p. 75) afirma que "Hay [...] una característica notable que ha emergido tanto a través del estudio de sistemas dinámicos como del análisis cualitativo de procesos no-lineales que son reducibles a modelos matemáticos. Progresivamente se ha puesto de manifiesto que fenómenos de muy diversa naturaleza, que pertenecen al dominio de diferentes disciplinas y que, desde el punto de vista de una descripción puramente fenomenológica, parecerían no tener nada en común, presentan una gran similitud en lo que respecta a su evolución temporal".

Este breve resumen de la teoría de los sistemas complejos que propone Rolando García es la base que le permite a este autor afirmar cómo es que "la intención de la obra [2000] fue poner de manifiesto el carácter empírico (pero no-empirista) de la epistemología genética, no sólo porque su fundamentación es empírica sino porque, a su vez, fundamenta una concepción conceptual y metodológica particular: la investigación interdisciplinaria de los sistemas complejos" (2006, p. 17). Investigación que es, precisamente, el propósito de esta segunda obra de García. En ella concibe que, dado que la realidad es compleja, la única manera de considerar aspectos particulares de un sistema (fenómeno, proceso o situación) es estudiándolo a partir de la interdisciplinariedad, ya que un sistema complejo es una totalidad organizada, constituida por elementos heterogéneos e inseparables que interactúan, por lo que no pueden ser estudiados aisladamente (2006, pp. 21, 32). 
La metodología que propone este autor parte entonces de investigar sistemas complejos así definidos, que incluyen aspectos físicos, biológicos, sociales, económicos, políticos e históricos $(2006$, pp. 47, 54) a partir de la conformación de un grupo multidisciplinario cuyos miembros compartan marcos epistémicos, conceptuales y metodológicos, que debe, para empezar, formular las preguntas de base sobre los problemas que se busca interpretar y resolver, incluyendo estudios anteriores relacionados con la problemática. Aquí es importante destacar que Rolando García hace hincapié en algo que es de sobresaliente importancia: el equipo es multidisciplinario y la interdisciplinariedad aparece en el proceso, ya que lo que es interdisciplinario es la metodología no el equipo de investigación, el cual es, simplemente, multidisciplinario ${ }^{7}$ (2006, p. 89).

La siguiente fase de la metodología consiste en caracterizar un sistema que involucre la problemática definida antes, incluyendo sus condiciones de contorno, que son las "interacciones... [o] flujos a través de los límites del sistema construido (límites geográficos o conceptuales)" (2006, p. 128). Aquí, con respecto al calificativo de "construido" que García aplica al sistema, vale la pena hacer hincapié en que la epistemología constructivista de este autor parte de que los "hechos" u "observables" con los que trabaja el equipo interdisciplinario son interpretaciones que hacen que "el hecho [sea] solidario de un sistema de conceptos (y "preconceptos") que corresponden a la experiencia previa del investigador y a su propia "ideología" (considerando este último término...como concepción del mundo)" (2006, p. 78). O, en citas del propio Rolando García (2006, p. 77), según Piaget, "No hay lectura pura de la experiencia" y, según Russell Hanson, "Todo observable está cargado de teoría".

A lo anterior seguiría el planteamiento de las hipótesis de trabajo, seguido de la identificación de la problemática por investigar en cada subsistema relevante, mediante investigaciones correspondientes a la disciplina o disciplinas involucradas en cada subsistema.

Posteriormente, se tendría que hacer una primera integración de los resultados obtenidos en la etapa anterior y la repetición de estas dos últimas fases para redefinir el sistema en su conjunto (integrado o reintegrado), hasta "llegar a una explicación

\footnotetext{
${ }^{7}$ No obstante lo anterior, sería objeto de otra discusión el hecho de que toda persona es efectivamente interdisciplinaria en alguna medida, toda vez que cada quien tiene competencias, poco o muy desarrolladas, en más de un campo del conocimiento o disciplina.
} 
coherente que dé cuenta de todos los hechos observados y responda a las preguntas que han ido surgiendo en el proceso descrito" (2006, p. 101).

Puede notarse en la anterior descripción de las fases de un estudio interdisciplinario que hay etapas de diferenciación y de integración que se corresponden con las reorganizaciones sucesivas que se dan en la construcción del conocimiento, tal como lo propone la epistemología constructivista.

Un proceso de investigación como el esbozado culmina con un diagnóstico sobre el sistema complejo que se estudia y le sigue, necesariamente, una investigación sobre el curso de acción que debe seguirse para resolver el o los problemas que el sistema presenta y que tiene analogías con la investigación diagnóstica, pero también diferencias. De éstas, las más notorias son que, en el caso de la investigación sobre el curso de acción por seguir, se trata, en primer lugar, de una investigación prospectiva y, en segundo término, el proceso incluye poner en práctica medidas correctivas que alteran al sistema, por lo que se hace necesario dar seguimiento a su evolución, conforme se implantan esas medidas, con el propósito de asegurar que se alcanzan los objetivos deseados.

Para terminar esta breve exposición de la postura sobre la complejidad desde las ciencias sociales, vale la pena anotar que, además de lo señalado como representativo de esta corriente, existen, además, lo que pudiera denominarse "aproximaciones disciplinarias", en las que se abordan diversas disciplinas sociales, desde las "ciencias de la complejidad". Existe abundante literatura sobre complejidad y psicología, complejidad y economía, complejidad y administración (pública y de empresas), complejidad y sociología, etc. Sin embargo, no se anotan mayores detalles sobre esto porque, aunque es de resaltar su existencia, no constituyen propiamente enfoques globales a la complejidad, como los tres analizados antes. Sólo se reitera aquí que en un trabajo posterior se analizará el tema de la relación entre la complejidad y la administración.

\section{Consideraciones finales}

Las tres aproximaciones a la complejidad que se han expuesto muy resumidamente coinciden en varias cuestiones. La primera de ellas, y quizá la más importante, es con respecto al análisis de sistemas complejos que, en los tres casos, tienen características comunes: se trata de conjuntos numerosos de elementos que interactúan 
y se autoorganizan, frecuentemente en forma de red, sin un controlador central y que, en ese proceso, producen conductas emergentes.

Estos sistemas complejos son abiertos: tienen intercambios con su medio ambiente, lo cual les permite mantenerse estables pero, al mismo tiempo, lejos del equilibrio, ya que operan con variaciones constantes, dentro de cierto rango. Tienen mecanismos que les permiten absorber las variaciones externas que los alteran y que, cuando rebasan cierto umbral o punto crítico, los hacen desestabilizarse para, después de un periodo caótico en el que se autorreorganizan, llegar a un nuevo periodo de estabilidad fuera del equilibrio; es decir, se adaptan.

Otro punto en el que coinciden los tres enfoques es la necesidad de abordar los sistemas complejos, o la realidad misma o aspectos particulares de ella, a partir de la interdisciplina, aunque, por otro lado, existen interpretaciones diversas sobre lo que "interdisciplinariedad" significa y, a la vez, se utilizan diferentes términos: el C3 y el Instituto Santa Fe hablan de "multidisciplina", en tanto que Melanie Mitchell y Rolando García se refieren a "interdisciplina".

En cuanto a las diferencias más notables entre las tres maneras de abordar la complejidad se encuentra la postura sobre si existe o no una teoría o ciencia de la complejidad. Por un lado, Rolando García afirma que lleva 30 años construyendo la Teoría de los Sistemas Complejos y, por su parte (aunque no es el único), Neil Johnson afirma que no se tiene aún una teoría de la complejidad completamente desarrollada.

Por otra parte, aunque, como se anotó anteriormente, las tres aproximaciones también coinciden en la existencia de los sistemas complejos, la tipificación que de ellos se hace desde las ciencias duras es muy distinta a la que propone Rolando García desde las ciencias sociales, al tiempo que Edgar Morin, aunque habla de sistemas y se puede colegir que se refiere a sistemas complejos, no los menciona específicamente.

De igual forma, parece que no existe un diálogo fluido entre los tres enfoques. Los diversos textos consultados sobre la complejidad vista desde las ciencias duras (Érdi, 2008; Mitchell, 2009; Gribbin, 2004; y Johnson, 2007) no mencionan para nada las propuestas que se hacen desde las perspectivas universal y social, ejemplificadas aquí con trabajos de Edgar Morin, de Fritjof Capra o de Rolando García, aun a pesar de que en el Instituto Santa Fe, quizá la organización más representativa de la primera corriente y de la cual forma parte Melanie Mitchell, se afirma, 
como se anotó antes, que busca "descubrir, comprender y comunicar los principios comunes fundamentales de los sistemas complejos físicos, computacionales, biológicos y sociales".

Además, las metodologías para abordar los sistemas complejos son muy distintas. La de las ciencias duras se inclina drásticamente hacia las matemáticas y la computación, mientras que la que se propone desde las ciencias sociales se basa en el trabajo interdisciplinario de un grupo multidisciplinario de especialistas que, aunque no excluye las matemáticas, no les da la misma importancia que la primera. Por su parte, Edgar Morin propone ir avanzando en la construcción del método (que está aún por definir) con lo que, a la vez, se irá también avanzando en la comprensión de la complejidad y en la resolución de problemas.

Otro punto en el que es clara la diferencia entre ciencias duras y sociales es el que se refiere a las aplicaciones del análisis. Desde las ciencias duras, las aplicaciones se dan en problemas muy específicos, que se pueden modelar y resolver, como se ha dicho, principalmente con métodos matemáticos o computacionales lo cual, incidentalmente, está dando lugar a importantes avances en muy diversas áreas. Las aplicaciones que se han dado en el área de las ciencias sociales, y que también han rendido frutos, consisten en abstraer "trozos" de la realidad, que son más amplios que los que se abordan desde las ciencias duras, y que incluyen aspectos tanto físicos, como biológicos, antropológicos y sociales.

Y existe, por otra parte, la propuesta que Edgar Morin plantea como la necesidad de abordar los problemas "pertinentes", fundamentales y globales y que involucran al sistema complejo que constituye el planeta Tierra como un todo. Y, sobre esto, hay cuando menos dos cuestiones vitales que es necesario considerar. Está, por un lado, la evidente imposibilidad actual de abordar problemas de envergadura mundial debido a la evidente falta de disposición de diversos países y de diversos sectores y actores sociales para intentar resolver problemas como la desigual distribución del ingreso al interior de cada país y entre países o la contaminación ambiental que son asuntos planetarios y, en segundo término, la imposibilidad técnica actual de resolver problemas de gran envergadura que, en su extremo, implicaría resolver todos los numerosos subsistemas que componen al Gran Sistema Mundial. Sin embargo, sobre este último punto, este ensayista coincide con lo que plantea Ray Kurzweil cuando afirma que la "inminente fusión de nuestro pensamiento biológico con la inteligencia no biológica que estamos creando" (Kurzweil, 2005 , p. 4) va a permitir la creación de computadoras, la re-creación de humanos y 
la interacción entre unas y otros que hará posible, cuando menos técnicamente, la resolución de problemas de envergadura planetaria, o aún mayores. En palabras de Kurzweil: "En última instancia, el universo completo quedará saturado con nuestra inteligencia. Éste es el destino del universo.” (Kurzweil, 2005, p. 29)

Las aproximaciones actuales a la complejidad constituyen un área de investigación que está en proceso de consolidación y que está dando lugar a una nueva comprensión del mundo y a avances y aplicaciones en muy distintos frentes, tanto en las ciencias duras, como en las ciencias sociales y las humanidades, al tiempo que se proponen traspasar las barreras disciplinarias y las que separan a estos tres campos del saber. Y, con respecto a la administración, como se mostrará en un trabajo posterior, ya existen abundantes trabajos tanto teóricos como prácticos que abordan su relación con la complejidad y que, también, están produciendo resultados tanto visibles como prometedores.

\section{Referencias}

Capra, Fritjof (2002). Las conexiones ocultas, implicaciones sociales, medioambientales, económicas y biológicas de una nueva visión del mundo. Barcelona: Anagrama.

Díaz Mata, Alfredo (1996). Caos, fractales, mercados de valores y triceversa. Contaduría y Administración, abril-junio (181).

Érdi, Péter, (2008). Complexity explained. Berlín: Springer.

García, Rolando (2000). El conocimiento en construcción, de las formulaciones de Jean Piaget a la teoría de los sistemas complejos. Barcelona: Gedisa.

- (2006). Sistemas complejos, conceptos, método y fundamentación epistemológica de la investigación interdisciplinaria. Barcelona: Gedisa.

Gribbin, John (2004). Deep simplicity, bringing order to chaos and complexity. Nueva York: Random House.

Johnson, Neil (2007). Simply complexity, a clear guide to complexity theory. Oxford: One World Publications. 
Köppen, Elke, Ricardo Mansilla y Pedro Miramontes (2005). La interdisciplina desde los sistemas complejos. Ciencias, julio-septiembre (79).

Kurzweil, Ray (2005). The singularity is near, when humans transcend biology. Nueva York: Penguin Books.

Leff, Enrique (2008). Discursos sustentables. México: Siglo XXI.

Mandelbrot, Benoit B. (1977). The fractal geometry of nature. Nueva York: W.H. Freeman and Company.

Mitchell, Melanie (2009). Complexity, a guided tour. Nueva York: Oxford University Press.

Morin, Edgar (2001). Los siete saberes necesarios para la educación del futuro. México: Dower.

(2007). Introducción al pensamiento complejo. Barcelona: Gedisa. (2006). El método I, la naturaleza de la naturaleza. $7^{\text {a }}$ edición, Barcelona: Gedisa.

— Emilio Roger y Raúl D. Motta (2006). Educar en la era planetaria. Barcelona: Gedisa.

Sotolongo Codina, Pedro Luis y Juan Jesús Delgado Díaz (2006) (Coordinador). La revolución contemporánea del saber y la complejidad social. Hacia unas ciencias sociales de nuevo tipo. Buenos Aires: CLACSO.

Páginas en Internet

http://ciuem.org/?q=node/5

http://css.csregistry.org/tiki-index.php www.amazon.com www.comdig.org www.ipcem.net www.jstor.org/pss/2640329

www.mcxapc.org www.nucleares.unam.mx/C3/?q=es/node/19 www.santafe.edu www.sld.cu/sitios/complejidad/index.php 